\title{
Síndrome constitucional por tumor carcinoide: afectación cardíaca característica
}

Leyre Sáenz de Pip
José Ramírez Bat
Laura Hurtado Ca
* Departamento de
** Departamento de
*** Departamento de
Recibido: 12/01/2020
Aceptado: 12/01/2020

En línea: 02/03/2020

Citar como: Sáenz de Pipaón-Ayala L, Ramírez-Batista J, Hurtado-Carrillo L, Ariño-Pérez de Zabalza I, Azcarate-Agüero PM. Síndrome constitucional por tumor carcinoide: afectación cardíaca característica. Rev Ecocar Pract (RETIC). 2020 (Mar); 3 (1): 45-48. doi: 10.37615/retic.v3n1a12.

Cite this as: Sáenz de Pipaón-Ayala L, Ramírez-Batista J, Hurtado-Carrillo L, Ariño-Pérez de Zabalza I, Azcarate-Agüero PM. Constitutional syndrome due to carcinoid tumor: characteristic cardiac involvement. Rev Ecocar Pract (RETIC). 2020 (Mar); 3 (1): 45-48. doi: 10.37615/retic.v3n1a12.

\section{Palabras clave}

$\triangleright$ Síndrome carcinoide

$\triangleright$ Tumor neuroendocrino

$\triangleright$ Valvulopatía adquirida

\section{Keywords}

$\triangleright$ Carcinoid syndrome

$\triangleright$ Neuroendocrine tumors

$\triangleright$ Acquired valvulopathy

\author{
Irene Ariño Pérez de Zabalza*** \\ Pedro María Azcárate Agüero**
}

\begin{tabular}{|l|}
\hline Correspondencia \\
\hline $\begin{array}{l}\text { Úrsula Vargas-Gómez } \\
\text { ursula.mvg@gmail.com }\end{array}$ \\
\hline
\end{tabular}

\begin{abstract}
RESUMEN
Los tumores carcinoides son tumores neuroendocrinos que derivan de células enterocromafines. La mayoría se localizan en el tracto gastrointestinal y los bronquios. El síndrome carcinoide se caracteriza por rubefacción de cara y tronco, diarrea, broncoespasmo y disnea. El síndrome carcinoide es una causa rara de valvulopatía adquirida, sin embargo, entre el 20-60\% de los pacientes presenta afectación cardíaca, siendo la causa de muerte más frecuente. Se desarrolla un caso de afectación pulmonar y tricúspide secundaria a este síndrome con revisión de la literatura del mismo.
\end{abstract}

ABSTRACT
Carcinoid tumors are neuroendocrine tumors arising from enterochromaffin cells. The most common localiza-
tion is the gastrointestinal tract and the bronchia. Carcinoid syndrome is characterized by flushing of the face
and trunk, diarrhea, bronchial spasm, and dyspnea. Carcinoid syndrome is a rare cause of acquired valvulo-
pathy, however, between 20-60\% of patients have heart involvement being the most common cause of death.
We present a case of tricuspid and pulmonary valvular disease secondary to this syndrome with review of the
literature.

\section{Presentación del caso}

Paciente varón de 78 años con antecedentes personales de hipertensión arterial, dislipidemia y deterioro cognitivo moderado. A nivel cardiovascular presentaba fibrilación auricular permanente diagnosticada desde hacía un año. Acude a urgencias por presentar deterioro del estado general, junto con anorexia, pérdida de peso, deposiciones diarreicas con sangre ocasionalmente, edemas en extremidades inferiores y aumento del perímetro abdominal.

En la exploración física las constantes vitales eran: presión arterial 140/75 mmHg, frecuencia cardíaca 95 lpm y saturación de $\mathrm{O}_{2}$ 90\% basal. Auscultación cardíaca: arrítmica, soplo holosistólico en foco tricúspide y otro soplo holodiastólico en foco pulmonar. Auscultación pulmonar: disminución marcada del murmullo vesicular fisiológico en base pulmonar derecha. Abdomen: blando, distendido, no doloroso a la palpación. Ascitis y hepatomegalia de dos traveses. Peristaltismo conservado y sin signos de irritación peritoneal. Extremidades inferiores: edemas con fóvea hasta raíz de muslos.
En la analítica se detectó, como hallazgos patológicos, anemia leve de perfil ferropénico (Hb 11,8 g/l, VCM 75,5f), elevación de transaminasas (GGT 202 U/l, FA UI/I 299), hipoalbuminemia (albúmina $3 \mathrm{~g} / \mathrm{dl}$ ), ferropenia (ferritina $50 \mathrm{ng} / \mathrm{dl}$, IsT 7\%), elevación de péptidos natriuréticos (NT-pro-BNP 1698 pg/ml) y CA-125 de $904 \mathrm{U} / \mathrm{ml}$. En la radiografía de tórax se observó cardiomegalia con derrame pleural derecho.

Se inició el estudio del síndrome constitucional realizando una body-TC (Figura 1 y Figura 2). En el tórax se objetivó derrame pleural derecho con atelectasia del pulmón subyacente y cardiomegalia. En el abdomen se observaron varias lesiones ocupantes de espacio (LOES) hepáticas con aumento de captación de contraste, que plantearon el diagnóstico diferencial de metástasis de neoplasia hipervascular frente a adenomas hepáticos múltiples. Además, las venas suprahepáticas mostraban signos congestivos y existía abundante líquido peritoneal.

Al mismo tiempo, y dados los signos de insuficiencia cardíaca, se solicitó una ecocardiografía que mostró un ventrículo izquierdo con fracción de eyección conservada con movimiento paradójico del septo interventricular en relación con la presencia de sobrecarga de volumen de cavidades derechas (Vídeo 1). 
El ventrículo derecho estaba moderadamente dilatado con FEVD conservada (Vídeo 2). La válvula tricúspide presentaba una insuficiencia severa por mecanismo de cierre valvular restrictivo. Los velos de esta válvula están engrosados y no coaptan en sístole (Vídeo 3 y Vídeo 4). La válvula pulmonar también presentaba una insuficiencia severa por mecanismo de cierre valvular restrictivo (Vídeo 5).

Dados los hallazgos de la TC y las características de la afectación de las válvulas tricúspide y pulmonar (sugestivas de afectación "carcinoide"), el estudio se dirigió a la búsqueda del tumor primario. Por ello se realizó un estudio de medicina nuclear: gammagrafía con pentreótido marcado con indio111 (estudio con gammacámara que detecta la captación del radiofármaco por los receptores de somatostatina), con el que se evidenció captación a nivel hepático y del intestino grueso confirmando la sospecha de tumor neuroendocrino carcinoide (Figura 3).

Además, se detectó en suero la presencia de cromogranina A (proteína presente en los gránulos de secreción neuroendocrina) y ácido-5-hidroxindolacético en orina de 24 horas (producto de degradación de serotonina, que es segregado por el tumor).

Para la filiación anatomopatológica de la neoformación se llevó a cabo una punción-aspiración con aguja gruesa de una de las lesiones hepáticas. En el informe histológico e inmunohistoquímico se describió una lesión neoplásica densamente celular en nidos sólidos con inmunorreactividad granular citoplasmática con la cromogranina y con la sinaptofisina (proteínas ambas asociadas a gránulos de secreción neuroendocrina). Se llegó así al diagnóstico de metástasis de tumor neuroendocrino, bien diferenciado (grado I), de origen intestinal.

Con este diagnóstico y dada la situación basal del paciente (con demencia moderada) se decidió realizar tratamiento conservador del tumor primario con octreótido (análogo de la somatostatina) para inhibir la secreción de sustancias neuroendocrinas, así como tratamiento farmacológico de la insuficiencia cardíaca derecha.

\section{Estudio por imagen}

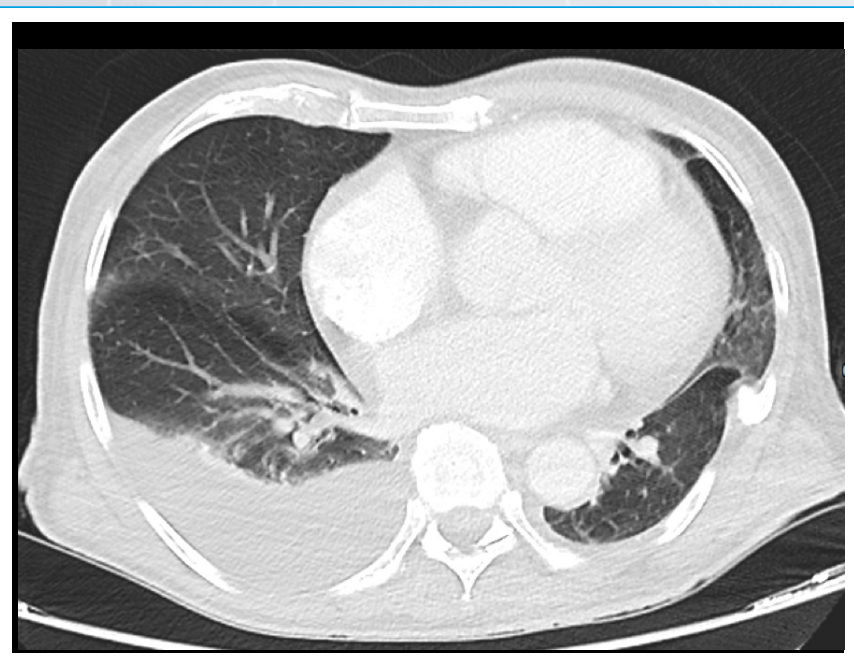

Figura 1. TC corte axial torácico a nivel cardíaco. Se observa derrame pleural derecho con atelectasia pasiva del parénquima adyacente. No hay imágenes nodulares o masas pulmonares. Cardiomegalia. No adenopatías mediastínicas, hiliares ni axilares

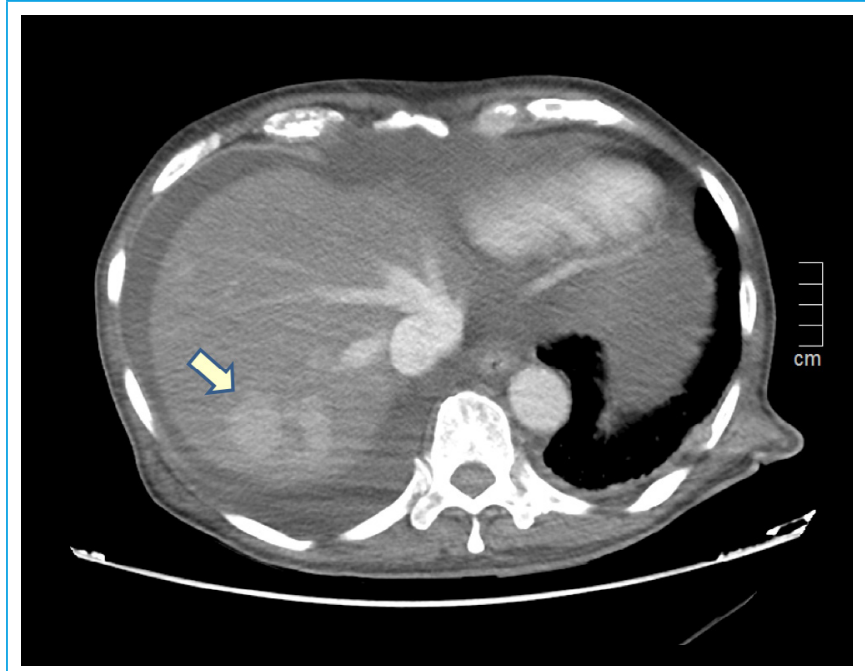

Figura 2. TC corte axial abdominal a nivel hepático. Sistema venoso suprahepático dilatado por insuficiencia cardíaca derecha. Varias LOES hepáticas hipercaptantes de contraste de forma homogénea (flecha), que plantean el diagnóstico diferencial entre metástasis de neoplasia hipervascular frente a adenomas hepáticos múltiples. Abundante líquido libre intraperitoneal perihepático, periesplénico, sin observar nódulos peritoneales. No adenopatías
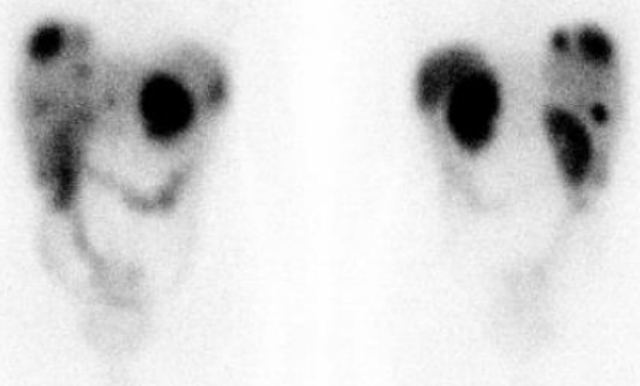

Figura 3. Gammagrafía con pentreótido marcado con indio111 (cortesía del Dr. Cabrera. Médico Especialista de Medicina Nuclear. Hospital San Pedro)

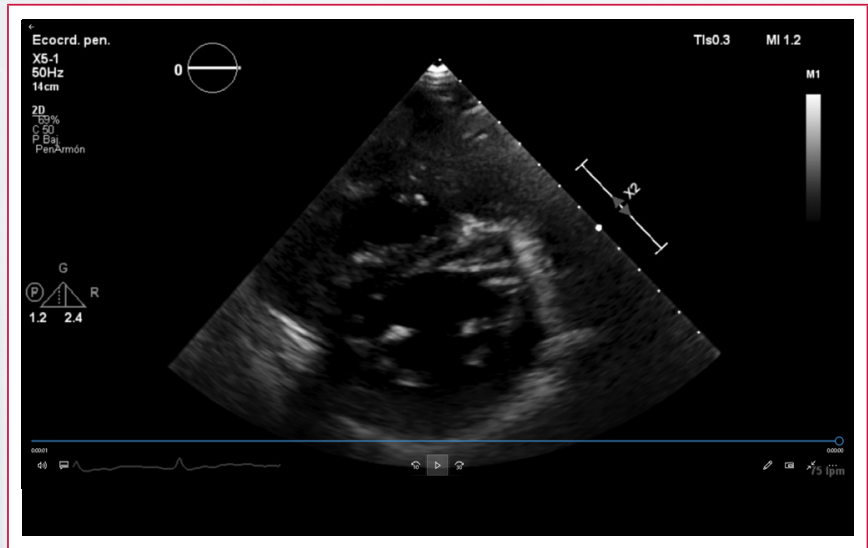

Vídeo 1. ETT eje corto. Se observa movimiento paradójico del septo interventricular en relación con la presencia de sobrecarga de volumen de cavidades derechas y un derrame pericárdico leve 


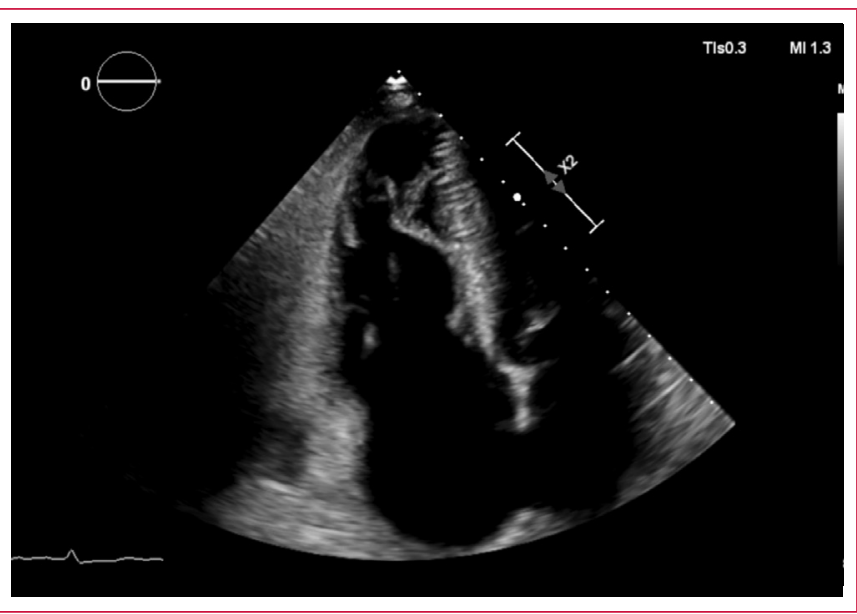

Vídeo 2. ETT apical de cuatro cámaras focalizada en el ventrículo derecho (VD). VD dilatado: DTD basal $50 \mathrm{~mm}$ con fracción de eyección conservada, TAPSE $17 \mathrm{~mm}$ y FAC 45\%. Aurícula derecha moderada-severamente dilatada

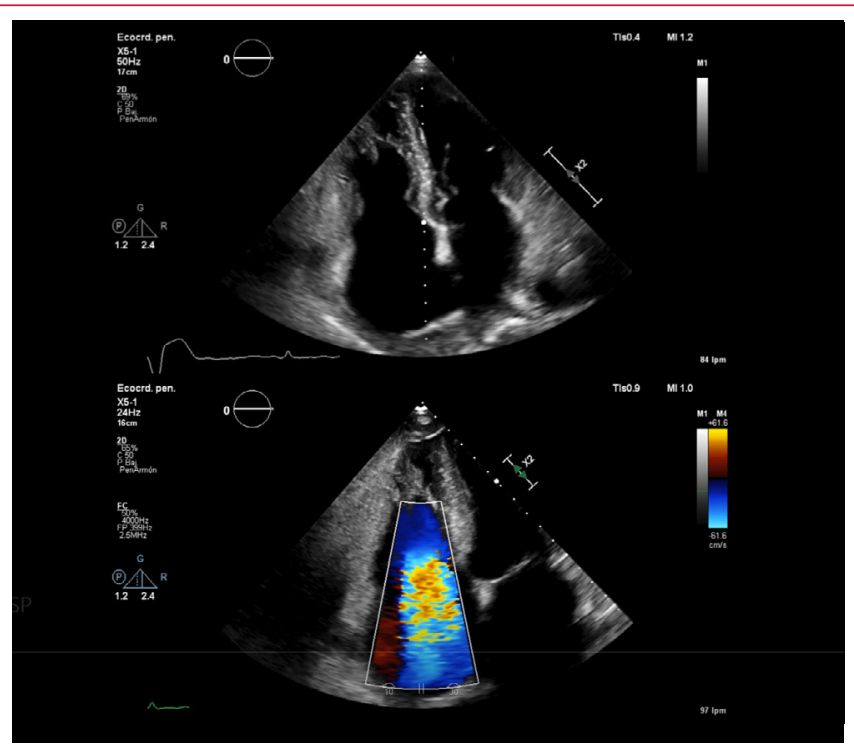

Vídeo 3. ETT apical de cuatro cámaras con y sin color. Arriba: los velos de esta válvula tricúspide están engrosados y no coaptan en sístole. Abajo: insuficiencia severa por mecanismo de cierre restrictivo

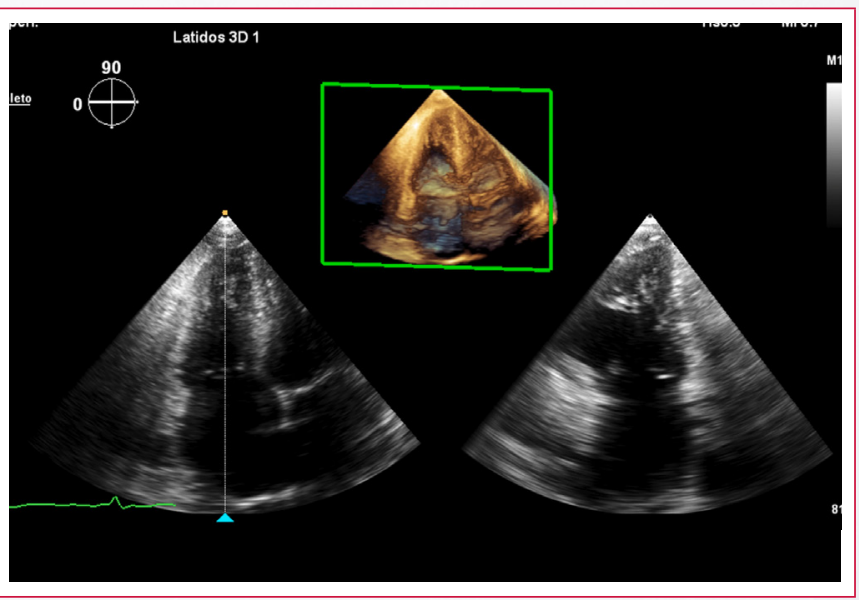

Vídeo 4. ETT apical tridimensional, detalle de la válvula tricúspide

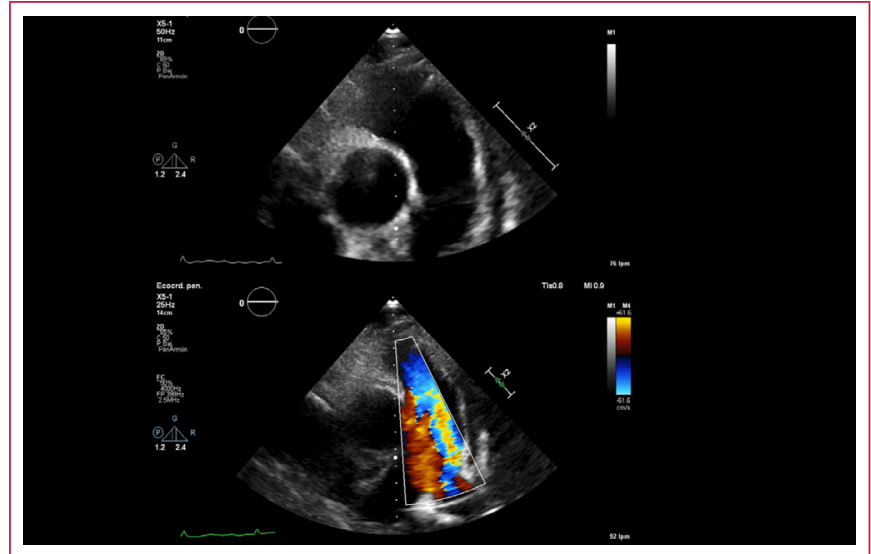

Vídeo 5. ETT plano grandes vasos, detalle de la válvula pulmonar. Arriba: los velos de la valvular pulmonar están engrosados y no coaptan en diástole. Abajo: insuficiencia pulmonar severa por el mismo mecanismo que la válvula tricúspide

\section{Discusión}

El síndrome carcinoide es un cuadro clínico paraneoplásico secundario a la liberación de sustancias vasoactivas por tumores neuroendocrinos. El síntoma más frecuente es el flushing facial, seguido de diarrea, dolor abdominal y broncoespasmo.

Entre el 20-60\% de los pacientes con tumores neuroendocrinos pueden presentar afectación cardíaca(1) (esta gran variabilidad depende en buena parte del grado de extensión y de la secreción de sustancias por el tumor). Aunque los mecanismos de la afectación cardíaca no están totalmente aclarados, se sabe que la producción de serotonina desempeña un papel destacado en el desarrollo de patología a nivel cardíaco. La afectación cardíaca se caracteriza por la aparición de fibrosis en las cámaras cardíacas derechas. Esta "preferencia" por el lado derecho del corazón se debe a que las sustancias vasoactivas son "limpiadas" en el pulmón, por lo que su concentración en cavidades izquierdas es mucho más baja. Los hallazgos típicos son engrosamiento y retracción tanto de las valvas como del aparato subvalvular, lo que impide la adecuada coaptación de los velos con la consiguiente regurgitación ${ }^{(2)}$. También pueden observarse alteraciones segmentarias de la contractilidad en el VD como consecuencia de fibrosis endocárdica. La regurgitación tricúspide es el hallazgo más frecuente y suele ser al menos moderada. La válvula pulmonar también se suele engrosar y retraer.

El adecuado reconocimiento de la afectación carcinoide a nivel cardíaco es fundamental. Por un lado, en pacientes no diagnosticados puede ser clave para llegar a un diagnóstico y tratamiento precoz. Por otro, la afectación cardíaca es el principal determinante pronóstico de los pacientes con tumores carcinoides, por lo que su manejo adecuado es decisivo.

El tratamiento con análogos de la somatostatina (octreótido y lanreótido) es necesario para controlar la producción de sustancias vasoactivas y detener la progresión de la afectación cardíaca. El tratamiento con diuréticos y restricción hidrosalina es la piedra angular del tratamiento sintomático de estos pacientes aunque, en muchos casos, puede no ser suficiente. Puesto que el fallo cardíaco derecho secundario a la valvulopatía tricúspide es la principal causa de muerte en estos pacientes, en algunos casos debe plantearse la sustitución valvular. La selección de pacientes candidatos y la elección del momento de la cirugía es controvertida ${ }^{(3)}$. El riesgo quirúrgico es, en general, elevado (oscila entre el 10$20 \%$ en centros experimentados), pero puede incrementarse aún más cuando el fallo del VD está establecido. 


\section{Conclusión}

La afectación cardíaca carcinoide es una entidad infrecuente, pero que conviene tener presente en el diagnóstico diferencial de la patología valvular derecha. Como se ha expuesto, su reconocimiento puede ser esencial tanto para el diagnóstico como para el pronóstico de los pacientes con tumores carcinoides ${ }^{(4)}$.

\section{Ideas para recordar}

- El síndrome carcinoide se caracteriza por rubefacción de cara y tronco, diarrea, broncoespasmo y disnea.

- La afectación cardíaca carcinoide es una entidad infrecuente, pero que conviene tener presente en el diagnóstico diferencial de la patología valvular derecha.
- Los hallazgos valvulares típicos son: engrosamiento y retracción tanto de las valvas como del aparato subvalvular, lo que impide la adecuada coaptación de los velos con la consiguiente regurgitación.

\section{Bibliografía}

1. Hassan SA, Banchs J, lliescu C, et al. Carcinoid heart disease. Heart 2017; 103 (19): 1488-1495.

2. Miyasaka R, Mehta A, Pettersson GB, et al. Carcinoid Tricuspid Valve Disease: Applications of Three Dimensional Transesophageal Echocardiography. Circ Cardiovasc Imaging 2019; 12: e009555.

3. Davar J, Connolly HM, Caplin ME, et al. Diagnosing and Managing Carcinoid Heart Disease in Patients With Neuroendocrine Tumors: An Expert Statement. J Am Coll Cardio/ 2017; 69: 1288-1304.

4. Dashwood A, Rahman A, Pavicic M. Carcinoid heart disease. Eur Heart $J$ $2015 ; 36: 2326$ 